\title{
Uso de Moodle para fortalecer competencias ciudadanas en estudiantes de fisioterapia: un diseño pretest-postest \\ Use of Moodle to strengthen citizenship competencies in physiotherapy students: a pretest-posttest design
}

Milena Boneth-Collantes, Jorge Salazar-Rodríguez, Adriana Angarita-Fonseca

Universidad de Santander (Colombia)

Resumen. Introducción: Moodle es una tecnología de información y comunicación de aprendizaje ampliamente utilizada en todo el mundo y se ha utilizado poco para fortalecer las competencias ciudadanas. Objetivo: Mejorar las competencias ciudadanas a través de una estrategia pedagógica en la plataforma Moodle en estudiantes registrados en los cursos formativos de práctica clínica/administrativa de fisioterapia de la Universidad de Santander, Bucaramanga, Colombia. Métodos: Se realizó un diseño pretest-postest. La intervención educativa consistió en una actividad pedagógica disponible en línea las 24 horas del día, en Moodle durante 10 semanas. Una muestra de 41 estudiantes cumplió con un cuestionario antes y después de la intervención educativa, que consistió en 25 preguntas distribuidas en cinco dimensiones: constitución política, multiperpectivismo, pensamiento sistémico y reflexivo, argumentación y ética. El puntaje total por dimensión osciló entre 0 a 100. Resultados: El puntaje promedio de conocimiento fue 60,8 \pm 12,9 en la prueba previa y 65,1 $\pm 15,4$ en la prueba posterior $(p=0,0665)$. Después de la intervención educativa, hubo cambios positivos en ocho de las 25 preguntas $(\mathrm{p}<0,005)$, tres de las cuales correspondían a la dimensión multiperspectivismo. En el análisis por subgrupos, se encontró que aquellos que obtuvieron un puntaje igual o menor a 60 en la evaluación inicial mejoraron significativamente sus conocimientos [pretest $=51.6$ (9.9), postest $=60(15.1)$, valor $\mathrm{p}=0,018]$. Conclusión: Una intervención educativa con Moodle aumentó el conocimiento de algunas competencias de ciudadanía entre los estudiantes de fisioterapia, especialmente en la dimensión multiperspectivismo.

Palabras Claves: Aprendizaje, Aula Extendida Moodle, Educación Basada en Competencias, Estudiantes, Evaluación educacional, Tecnología.

Summary. Introduction: Moodle is a learning information and communication technology widely used worldwide and has been little used to strengthen citizenship competencies. Objective:To improve citizenship competencies through a pedagogical strategy in the Moodle platform in students registered in the training courses of clinical/administrative physiotherapy practice at the University of Santander, Bucaramanga, Colombia. Methods: A pretest-posttest design was carried out. The educational intervention consisted of a pedagogical activity available online 24 hours a day in Moodle for ten weeks. A sample of 41 students completed a questionnaire before and after the educational intervention, which consisted of 25 questions distributed in five dimensions: political constitution, multiperspective, systemic and reflective thinking, argumentation, and ethics. The total score per dimension ranged from 0 to 100 . Results: The average knowledge score was $60.8 \pm 12.9$ in the pretest and $65.1 \pm 15.4$ in the posttest $(p=0.0665)$. After the educational intervention, there were positive changes in eight of the 25 questions $(p<0.005)$, three of which corresponded to the multiperspective dimension. In the analysis by subgroups, it was found that those who obtained a score equal to or less than 60 in the initial evaluation significantly improved their knowledge [pretest $=51.6(9.9)$, posttest $=60(15.1)$, p-value $=0.018$ ]. Conclusion: A educational intervention with Moodle increased the knowledge of some citizenship competencies among physiotherapy students, especially in the multi-perspective dimension.

Keywords: Learning, Moodle Extended Classroom, Competency-Based Education, Students, Educational Assessment, Technology.

\section{Introducción}

Los procesos de enseñanza-aprendizaje en la educación superior implican tanto el conocimiento de las competencias básicas, científicas y tecnológicas propias de su disciplina, como de las competencias ciudadanas que garanticen la formación integral en todas las dimensiones del ser, en coherencia con los retos actuales de desarrollo social, económico y productivo del país, que permita responder con pertinencia a las necesidades del

Fecha recepción: 02-08-20. Fecha de aceptación: 05-10-21

Milena Boneth

mi.boneth@mail.udes.edu.co entorno nacional, regional, local y global. (Ministerio de Educación Nacional, 2010).

Pérez \& Rodríguez (2016), realizaron un estudio para evaluar la adquisición de competencias ciudadanas en universitarios, los resultados demostraron que el uso de estas metodologías se asocia con un mayor rendimiento académico; adicionalmente profesores y estudiantes consideraron como exitoso el desarrollo del proyecto y percibieron las actividades ejecutadas como satisfactorias y útiles profesionalmente.

Las competencias ciudadanas facilitan la comprensión de la realidad social, la cooperación, la convivencia y la participación responsable en la construcción de una sociedad ética y democrática, de tal forma que el ciudadano demuestre una apropiación de su entorno y una 
participación social activa. (Chaux, 2004). En Colombia, actualmente se aplica el modelo educativo de formación por competencias, siendo una estrategia que le facilita al estudiante la comprensión del medio en el que se desenvuelve, para dar solución a problemas en diferentes contextos en el ámbito laboral y cotidiano, así como adquirir actitudes y aptitudes en el saber conocer, saber hacer y saber ser. (Betancourt, et al., 2020).

En el país, las áreas evaluadas en competencias ciudadanas son las contempladas en las pruebas de Estado de la Educación Superior e incluyen las dimensiones de Constitución Política; Multiperspectivismo; Pensamiento Sistémico y Reflexivo; Análisis de Perspectivas y Argumentación y Ética (Ministerio de Educación Nacional, 2010). Dentro de los resultados del examen de estado en estas competencias, el programa de Fisioterapia UDES muestra que en las pruebas SABER PRO de los años 2012, 2013 y 2014 los estudiantes obtuvieron puntajes por debajo del promedio nacional en competencias ciudadanas, siendo en el año 2012 la diferencia de promedios de 0,81 (Nacional: 10,13 \pm 0,67; fisioterapia UDES: 9,32 $\pm 0,75)$, en 2013 de 0,48 (Nacional: 10,11 \pm 1,02; fisioterapia UDES: 9,63, $\pm 0,72$ ) y en 2014 de 0,6 (Nacional: 10,09 $\pm 0,69$; fisioterapia UDES: 9,49, $\pm 0,77$ ). (Ministerio de Educación, 2014) Esto sugiere una posible deficiencia en la apropiación de conocimiento y aprendizaje asimilado por los alumnos durante su proceso de formación en relación con estas competencias.

De otra parte, el uso de los ambientes virtuales de aprendizaje como herramienta pedagógica para fomentar los procesos de enseñanza-aprendizaje en los estudiantes ha tomado fuerza con el transcurso del tiempo; debido en parte al desarrollo de internet y sus aplicaciones; razón por la cual, las instituciones de educación superior aumentaron la necesidad de transversalizar la virtualidad en los procesos de aprendizaje (Oproiu, 2015). En el estudio publicado por Oproiu (2015) los estudiantes manifestaron que el uso de este tipo de metodología como el caso de la plataforma Moodle aumentaba la eficiencia de los procesos de enseñanza, teniendo una percepción positiva en el $57 \%$ de los sujetos.

Una de las tecnologías más populares en la actualidad para dar soporte a la enseñanza como gestión de aprendizaje es la plataforma Moodle, la cual presenta código abierto con licencia libre que parte de un enfoque pedagógico social-constructivista, y busca fomentar la participación de los estudiantes y docentes, en donde los conocimientos orientados por el profesor no son la única fuente de información; ya que interactúan con los conocimientos del estudiante propiciando así, espacios de discusión y diálogo en el que los educandos reafirmen y reconstruyan su propios saberes. De igual manera, la arquitectura y herramientas de esta aplicación son adecuadas para clases en línea, complementando el aprendizaje presencial (Chaparro, Iglesias, \& Pascual, 2010; Ortiz, 2015).

En un estudio realizado por Cordero y Caballero (2015), la mayoría de los estudiantes encuestados sobre el apoyo de Moodle en los cursos o asignaturas presenciales, la consideró muy útil con un 50\%, útil $45,37 \%$, un $3,70 \%$ se manifestaron indiferentes, un $0,93 \%$ como poco útil y ningún estudiante lo consideró inútil. Moodle es la plataforma más utilizada con más de 37 millones de usuarios, y se ha aplicado en la construcción de más de 48198 sitios registrados en 212 países. (Castillo, Larios, \& García, 2010; Lamas, Massié, \& Quero, 2010).

El uso de las Tecnologías de la Información y la Comunicación que permiten combinar entornos presenciales y virtuales de aprendizaje ha aumentado progresivamente dentro de la práctica educativa, haciéndose prioritario desarrollar investigaciones que evalúen el valor agregado de las actividades de enseñanza asincrónicas en los modelos de enseñanza tradicionales impartidos de forma presencial. (García \& Pineda, 2010).

Lo anterior, evidencia la necesidad de realizar estudios que permitan determinar los cambios en los conocimientos sobre competencias ciudadanas en estudiantes universitarios. Este estudio tiene como objetivo mejorar las competencias ciudadanas por medio de la plataforma Moodle en estudiantes registrados que cursan práctica clínica/administrativa en fisioterapia. La hipótesis de los autores es que el uso de la plataforma Moodle incrementará el conocimiento de competencias ciudadanas en estos estudiantes.

\section{Material y método}

Se utilizó un diseño pretest-postest. Se evaluaron los cambios en los conocimientos sobre competencias ciudadanas antes y después del desarrollo de la estrategia pedagógica en la plataforma Moodle. En este caso, se contó con un solo grupo de estudiantes, sin grupo control y con mediciones pre y post intervención (Manterola \& Otzen, 2015). El estudio fue aprobado por el comité de investigaciones de la Maestría en Gestión de la Tecnología Educativa de la Universidad de Santander, Bucaramanga, Colombia.

\section{Participantes}


La muestra fue seleccionada por conveniencia. Estudiantes $(n=42)$ de los cursos de último año de Prácticas Formativas de Fisioterapia fueron invitados a participar. La muestra analizada quedó conformada por 41 participantes dado que un estudiante canceló el semestre. Las características de los participantes se muestran en la Tabla 1. El 92.85\% pertenecía al sexo femenino, el 90.47\% eran solteros (as). El porcentaje más alto de nivel socioeconómico correspondió a nivel tres con un $38.09 \%$ y el nivel con poca población fue uno, con el $4.76 \%$. El 2.39\% $(n=1)$ no poseía computador propio. El 73.8\% utilizaba el computador de cinco a siete días a la semana. El 88.1\% ya había utilizado la plataforma Moodle en asignaturas ya culminadas.

\begin{tabular}{|c|c|}
\hline Tabla 1 & Características generales de la población de estudio \\
\hline Características & $\%$ \\
\hline \multicolumn{2}{|l|}{ Edad } \\
\hline 19-22 años & 52.4 \\
\hline 23-26 años & 38.1 \\
\hline 27 o más & 9.5 \\
\hline \multicolumn{2}{|l|}{ Sexo } \\
\hline \multirow{2}{*}{\multicolumn{2}{|c|}{ Estado Civil }} \\
\hline & \\
\hline Solteros/as & 90.5 \\
\hline Casado/a & 4.7 \\
\hline Unión Libre & 2.4 \\
\hline Separado & 2.4 \\
\hline \multicolumn{2}{|l|}{ Estrato Socioeconómico } \\
\hline Nivel 1 & 4.7 \\
\hline Nivel 2 & 19.0 \\
\hline Nivel 3 & 38.1 \\
\hline Nivel 4 & 33.3 \\
\hline Nivel 5 & 11.9 \\
\hline \multicolumn{2}{|l|}{ Semestre Académico } \\
\hline 6 & 28.6 \\
\hline 7 & 28.6 \\
\hline 8 & 23.8 \\
\hline 9 & 1.0 \\
\hline Computador propio & 97.6 \\
\hline \multicolumn{2}{|l|}{ Frecuencia semanal de uso computador } \\
\hline Un día & 7.1 \\
\hline Entre 1 menos 3 días & 2.44 \\
\hline 3-5 días & 16.6 \\
\hline 5-7 días & 73.8 \\
\hline \multicolumn{2}{|l|}{ Tiempo diario frente a un computador } \\
\hline 1-2 horas & 9.5 \\
\hline 3-5 horas & 40.5 \\
\hline$>5$ horas & 50.0 \\
\hline Uso de la plataforma MOODLE en otras asignaturas & 88.1 \\
\hline
\end{tabular}

La duración de las fases fue de 16 semanas. Con relación a la intervención educativa consistió en 64 horas sobre los conocimientos de competencias ciudadanas distribuidas en 10 semanas, contando con la aceptación por parte de los participantes en el estudio, previa firma de consentimiento informado.

El estudio tuvo cinco fases.

Fase 1: Fase de Diseño y Capacitación a docentes. Se inició con una capacitación por parte del Departamento de Desarrollo Docente de la UDES, sobre el aula extendida de la Plataforma Moodle a los docentes que orientaban las asignaturas o cursos de prácticas formativas. Estas capacitaciones se realizaron en las aulas de informática de la Universidad.

Fase 2: Revisión Bibliográfica. Se realizó una revisión bibliográfica sobre estrategias pedagógicas con enfoque constructivista, y se escogieron las herramientas como foros, mapas conceptuales, ensayos y cuadros comparativos. Igualmente, todo lo concerniente a referentes bibliográficos sobre los temas a evaluar en Competencias Ciudadanas.

Fase 3: Capacitación a estudiantes. Se inició con una socialización a la población objeto sobre los objetivos del estudio y la participación de ellos en este; se firmó el consentimiento informado. Posteriormente por parte de los dos docentes de la investigación se desarrolló la capacitación sobre aula extendida de la Plataforma Moodle con una duración de cuatro horas en un solo encuentro; teniendo en cuenta que los estudiantes ya habían utilizado la plataforma en otras asignaturas o cursos del plan de estudio. El espacio utilizado fueron dos aulas de informática de la Universidad con una capacidad cada una de 25 estudiantes.

Fase 4: Evaluación inicial. Se diligenciaron los cuestionarios generales y de conocimientos sobre competencias ciudadanas antes de la aplicación de la estrategia pedagógica. La duración de estas primeras 4 fases fueron de 6 semanas.

Fase 5: Diseño e Implementación de la Estrategia Pedagógica. Se diseñaron las cuatro actividades dentro de la estrategia pedagógica seleccionada a desarrollar por los estudiantes, las cuales consistieron en cuatro actividades (Foro, cuadro comparativo, mapa conceptual y ensayo) disponibles en línea las 24 horas del día, los siete días de la semana en Moodle durante las últimas 10 semanas. (Tabla 2)

El foro, es una de las herramientas imprescindibles en las que se basa el trabajo colaborativo en un proceso de enseñanza aprendizaje, contempla debates y discusiones sobre un tema en particular donde el estudiante debe demostrar su capacidad de comprensión, análisis, reflexión y argumentación a través de sus aportes, generando habilidades cognitivas de orden superior; el cuadro comparativo permite identificar las diferencias y semejanzas de dos o más conceptos, permitiendo que el educando emita un juicio de valor como conclusión; el ensayo, requiere a su vez la consulta de diversas fuentes bibliográficas para fundamentar las ideas expuestas, por lo que estimula el pensamiento crítico, la metacognición y la habilidad de la comunicación escrita y por último el mapa conceptual, consiste en una representación gráfica de conceptos donde se promueve el pensamiento lógico, logra nuevos conocimientos y organización del pensamiento en el estudiante. (Pérez, Álvarez, García, Pascual, \& Fombona, 2004). 


\begin{tabular}{|c|c|c|c|c|c|c|}
\hline \multicolumn{7}{|c|}{ Estrategia Pedagógica } \\
\hline Semana & Competencia & Dimensión & Temas / subtemas & $\begin{array}{l}\text { Estrategias } \\
\text { metodológicas }\end{array}$ & Descripción de actividad & Indicador de logro \\
\hline 6-7 (12 horas) & $\begin{array}{l}\text { Que el estudiante identifique los } \\
\text { derechos y deberes que se tienen } \\
\text { como ciudadanos expresados en la } \\
\text { constitución política de Colombia. }\end{array}$ & Constitución Política & $\begin{array}{l}\text { Derechos, garantías y } \\
\text { deberes propuestos en } \\
\text { la Constitución } \\
\text { Política }\end{array}$ & $\begin{array}{l}\text { Foro. Lectura } \\
\text { previa: } \\
\text { Constitución } \\
\text { política: Titulo II, } \\
\text { derechos, } \\
\text { garantías y } \\
\text { deberes. }\end{array}$ & $\begin{array}{l}\text { Crear grupos de discusión con el docente orientador } \\
\text { como mediador de la actividad con una pregunta } \\
\text { orientadora: Todas las personas nacen libres } \\
\text { e iguales ante la ley, recibiendo el mismo trato y derechos } \\
\text { sin ningún tipo de discriminación. ¿Cree usted que se } \\
\text { cumple este enunciado de la constitución política en } \\
\text { todos los ámbitos? }\end{array}$ & $\begin{array}{l}\text { El aporte expuesto por el } \\
\text { estudiante presenta capacidad de } \\
\text { síntesis, análisis y coherencia. } \\
\text { sUtiliza correctamente las reglas } \\
\text { ortográficas. }\end{array}$ \\
\hline $8-9$ (12 horas) & $\begin{array}{l}\text { Que el estudiante establezca las } \\
\text { diferencias y semejanzas entre los } \\
\text { sistemas políticos y participación } \\
\text { ciudadana. }\end{array}$ & $\begin{array}{l}\text { Pensamiento } \\
\text { Sistémico y Reflexiv }\end{array}$ & $\begin{array}{l}\text { Responsabilidad } \\
\text { democrática y } \\
\text { participación } \\
\text { ciudadana. }\end{array}$ & $\begin{array}{l}\text { Cuadro } \\
\text { comparativo }\end{array}$ & $\begin{array}{l}\text { Previa lectura sobre el tema: Sistemas políticos y } \\
\text { participación ciudadana el estudiante debe realizar un } \\
\text { cuadro comparativo plasmando en las diferencias y } \\
\text { semejanzas, para que pueda identificar las características } \\
\text { del tema abordado con el fin de ser capaz posteriormente } \\
\text { de emitir un juicio de valor. }\end{array}$ & $\begin{array}{l}\text { Describe y compara los expuesto } \\
\text { en los sistemas políticos y } \\
\text { participación ciudadana a partir de } \\
\text { lo cual pueda plantear un juicio de } \\
\text { evalor. }\end{array}$ \\
\hline $\begin{array}{l}10-12(20 \\
\text { horas })\end{array}$ & $\begin{array}{l}\text { Que el estudiante sea capaz de } \\
\text { comprender las diferentes funciones } \\
\text { y elementos propios en los } \\
\text { órganos/y/o estructuras del estado }\end{array}$ & $\begin{array}{l}\text { Multiperspectivismo } \\
\text { /Análisis de } \\
\text { Perspectivas y } \\
\text { Argumentación }\end{array}$ & $\begin{array}{l}\text { Estructuras del estado: } \\
\text { Ramas Judicial, } \\
\text { Ejecutiva y Legislativa }\end{array}$ & & $\begin{array}{l}\text { Hacer una lectura analítica de las estructuras u órganos } \\
\text { del estado que aparecen en la constitución política } \\
\text { colombiana en el título v de la organización del estado } \\
\text { capítulo } 1 \text { de la estructura del estado }\end{array}$ & $\begin{array}{l}\text { Organiza todos los conceptos } \\
\text { estableciendo las relaciones y } \\
\text { jerarquías, presenta un producto de } \\
\text { excelente calidad. }\end{array}$ \\
\hline $\begin{array}{l}13-15 \text { (20 } \\
\text { horas) }\end{array}$ & $\begin{array}{l}\text { Que el estudiante sea capaz de } \\
\text { aplicar el concepto de ética en su } \\
\text { quehacer profesional. }\end{array}$ & Ética & $\begin{array}{l}\text { Ética y Derechos } \\
\text { Humanos }\end{array}$ & Ensayo & $\begin{array}{l}\text { Realizar una lectura previa del artículo: Vanegas G. José } \\
\text { H. Ética y Derechos Humanos en el Marco de la } \\
\text { constitución Política de Colombia de 1991. Universidad } \\
\text { Autónoma de Manizales. 2010. ISSN 1794-2918. } \\
\text { Posteriormente realizar un ensayo mínimo de } 3 \text { hojas y } \\
\text { máximo de } 5 \text { donde se evidencie su aporte personal, con } \\
\text { soporte bibliográfico actualizado. }\end{array}$ & $\begin{array}{l}\text { Presenta con claridad los conceptos } \\
\text { que sustentan la idea central, } \\
\text { utiliza correctamente las reglas } \\
\text { ortográficas, soportes } \\
\text { bibliográficos actualizados y } \\
\text { expone con coherencia su aporte } \\
\text { personal. }\end{array}$ \\
\hline
\end{tabular}

Los contenidos temáticos que se abordaron durante la intervención fueron las cinco dimensiones de competencias ciudadanas, Constitución Política; Multiperspectivismo; Pensamiento Sistémico y Reflexivo; Análisis de Perspectivas y Argumentación y Ética con el fin de fortalecer los conocimientos en competencias ciudadanas.

Fase 6: Evaluación Final. Posterior a la realización de la estrategia pedagógica implementadas en el Aula Extendida de Moodle, se realizó la evaluación final, con el mismo cuestionario de competencias ciudadanas aplicado al inicio, pero cambiando el orden de las preguntas y de las respuestas.

\section{Cuestionarios}

Los instrumentos utilizados fueron un cuestionario general y el cuestionario de conocimientos de competencias ciudadanas desarrollado por los autores para este proyecto. El cuestionario general indagó la edad, sexo, estado civil, semestre académico, estrato socioeconómico; tenencia del computador, horas semanales del uso de internet, tiempo diario del uso de internet, internet en casa, y el previo uso de la plataforma Moodle. El cuestionario de competencias ciudadanas constó de 25 preguntas de selección múltiple con cuatro opciones de respuesta, el cual fue creado por los autores e incluyó cinco dimensiones: Constitución Política $(n=7)$, Multiperspectivismo $(n=4)$; Pensamiento Sistémico y Reflexivo $(n=5)$; Análisis de Perspectivas y Argumentación $(n=2)$ y Ética $(n=7)$. El puntaje total y por dimensión osciló entre 0 y 100.

\section{Análisis Estadístico}

En el análisis descriptivo, las variables cualitativas se presentaron en frecuencias absolutas y relativas. Los puntajes se presentaron en promedio y desviación estándar cuando las variables se ajustaron a una distribución normal, de lo contrario se presentaron medianas, percentil 25 (P25) y percentil 75 (P75).

Para cada pregunta se comparó el porcentaje de respuestas correctas e incorrectas antes y después de la participación en Moodle mediante la prueba McNemar o prueba exacta de McNemar para datos pareados. Se consideró un cambio positivo si la respuesta de los estudiantes fue incorrecta en el pretest y correcta en el postest. Se consideró un cambio negativo si la respuesta fue correcta en el pretest e incorrecta en el postest. La distribución de cambios positivos, cambios negativos, respuestas correctas en ambas evaluaciones y respuestas incorrectas en ambas evaluaciones se presentó para cada pregunta.

Se realizó la comparación de los puntajes iniciales y finales totales y por dimensiones. Para ello, se utilizaron dos pruebas estadísticas. 1) La prueba $t$ de student para datos pareados en las dimensiones Constitución Política, análisis de perspectivas y argumentación, ética y el puntaje total, dado que los datos tuvieron una distribución normal. 2) La prueba rango con signos de Wilcoxon para datos pareados se aplicó en las dimensiones Multiperspectivismo, y pensamiento sistémico y reflexivo puesto que los datos no siguieron una distribución normal.

Para el análisis por subgrupos se creó una variable categórica usando como punto de corte la mediana del puntaje inicial (60), así dos grupos se crearon $(<=60$ y $>60$ ). El puntaje pretest y postest se comparó en cada una de las categorías de las variables sociodemográficas y de la nueva variable categórica puntaje inicial, para 
esto se usó la prueba t de student para datos pareados dado que los puntajes finales tuvieron una distribución normal. Adicionalmente, se realizaron análisis de regresión lineal simple y múltiple, en los cuales la diferencia pre-post fue la variable dependiente. En el modelo final se excluyó la variable computador propio por insuficiente tamaño de muestra en la categoría no. El análisis de ajuste de modelo se realizó con el test de especificación del modelo linktest.

El nivel de significancia usado fue 0.05. El análisis se realizó en el Software Stata 16.1

\section{Resultados}

En la Tabla 3, en la Dimensión Constitución Política se encontró un cambio positivo significativo en la pregunta relacionada con funciones de la rama ejecutiva del Poder Público (51\%, n=21) y con funciones de los representantes en la Cámara de Representantes del Departamento $(34.1 \%, \mathrm{n}=14)$. El puntaje total de esta dimensión no se incrementó después de la intervención educativa (Tabla 3. Figura 1).

En la Tabla 4 se observan cambios positivos y significativos en tres de los cuatros preguntas de la dimensión

Tabla 3

\begin{tabular}{|c|c|c|c|c|c|}
\hline No. de pregunta & $+a-$ & $-a+$ & ++ & $\ldots$ & Valor de $\mathrm{p}$ \\
\hline 1 & 12 & 0 & 23 & 6 & $.0005^{1}$ \\
\hline 2 & 5 & 8 & 25 & 3 & $.5811^{1}$ \\
\hline 8 & 6 & 21 & 10 & 4 & $.0059^{1}$ \\
\hline 9 & 12 & 8 & 18 & 3 & $.5034^{1}$ \\
\hline 10 & 2 & 14 & 24 & 1 & $.0042^{1}$ \\
\hline 11 & 10 & 6 & 21 & 4 & $.4545^{1}$ \\
\hline $\begin{array}{l}\text { Puntaje Promedio } \pm \\
\text { Desviación estándar }\end{array}$ & Pretest & $68.3 \pm 18.6$ & Postest & $72.4 \pm 24.6$ & $.3870^{2}$ \\
\hline $\begin{array}{l}+ \text { a }- \text { Preguntas contes } \\
-\mathrm{a} \text { +Preguntas contes } \\
++ \text { Preguntas contest } \\
\text { - Preguntas contestad } \\
{ }^{1} \text { Prueba Exacta de Mc } \\
{ }^{2} \text { Prueba t-test para da }\end{array}$ & $\begin{array}{l}\text { adas corr } \\
\text { das incor } \\
\text { las corre } \\
\text { sincorre } \\
\text { femar } \\
\text { s paread }\end{array}$ & $\begin{array}{l}\text { amente en pre } \\
\text { tamente en p } \\
\text { mente en pret } \\
\text { mente en pret }\end{array}$ & $\begin{array}{l}\text { st e incor } \\
\text { test y corr } \\
\text { t y postest } \\
\text { t y postest }\end{array}$ & $\begin{array}{l}\text { tamente en el } \\
\text { amente en el }\end{array}$ & \\
\hline
\end{tabular}
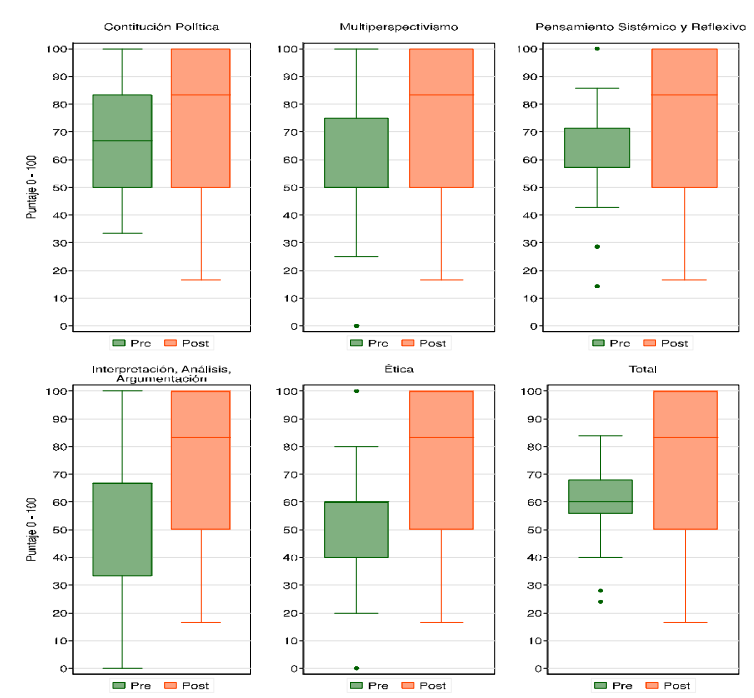

Figura 1. Puntaje pre y posttest según dimensión evaluada y total $(\mathrm{n}=41)$. Pre: Pretest. Evaluación Inicial Post: Postest. Evaluación Final
Multiperspectivismo, a saber: adopción de niños por parejas del mismo sexo $(31.7 \%, \mathrm{n}=13)$, prohibición del coleo $(35.6 \%, \mathrm{n}=15)$ y limitación diaria del número de carros particulares a circular $(43.9 \%, \mathrm{n}=18)$. El puntaje total de la dimensión Multiperspectivismo incrementó significativamente después de la intervención educativa $(\mathrm{p}=.001)$ (Tabla 4. Figura 1).

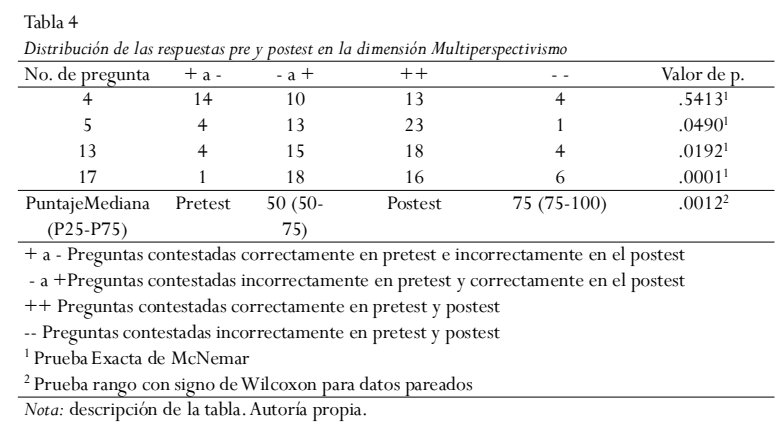

La Tabla 5 muestra que en la dimensión pensamiento sistémico y reflexivo, se observó un cambio positivo en dos de las siete preguntas que componen esta dimensión. Una pregunta estaba relacionada con el proceso de configuración histórica del sistema político colombiano $(46.3 \% \mathrm{n}=19)$ y otra sobre el trato desventajoso de los afroamericanos $(41.5 \%, n=17)$. No hubo cambios significativos en el puntaje de esta dimensión después de la intervención educativa (Tabla 5. Figura $1)$.

Tabla 5

\begin{tabular}{|c|c|c|c|c|c|}
\hline no. de pregunta & $+a-$ & $-a+$ & ++ & $\cdots$ & Valor de $\mathrm{p}$. \\
\hline 6 & 7 & 2 & 32 & 0 & $.1797^{1}$ \\
\hline 7 & 2 & 8 & 28 & 3 & .1094' \\
\hline 15 & 24 & 1 & 12 & 4 & $.1000^{1}$ \\
\hline 16 & 17 & 6 & 9 & 9 & $.0218^{2}$ \\
\hline 21 & 4 & 19 & 6 & 12 & .0026 ${ }^{1}$ \\
\hline 22 & 4 & 17 & 11 & 9 & . $0072^{1}$ \\
\hline 25 & 17 & 5 & 9 & 10 & $.0105^{2}$ \\
\hline $\begin{array}{c}\text { Puntaje } \\
\text { Mediana (P25-P75) }\end{array}$ & Pretest & $57.1(57.1-71.4)$ & Postest & $57.1(42.9-71.4)$ & $.1195^{3}$ \\
\hline $\begin{array}{l}+ \text { a - Preguntas con } \\
- \text { a }+ \text { Preguntas con } \\
++ \text { Preguntas cont } \\
-- \text { Preguntas contes } \\
{ }^{1} \text { Prueba Exacta de } 1 \\
{ }^{2} \text { Prueba de McNem } \\
{ }^{3} \text { Prueba t-test para }\end{array}$ & $\begin{array}{l}\text { tadas cor } \\
\text { tadas inc } \\
\text { adas corr } \\
\text { as incorr } \\
\text { Nemar } \\
\text { tos parea }\end{array}$ & $\begin{array}{l}\text { ectamente en prete } \\
\text { rectamente en pre } \\
\text { tamente en pretes } \\
\text { tamente en pretes }\end{array}$ & $\begin{array}{l}\text { e incorr } \\
\text { st y corr } \\
\text { y postest } \\
\text { y postest }\end{array}$ & $\begin{array}{l}\text { tamente en el post } \\
\text { tamente en el post }\end{array}$ & \\
\hline
\end{tabular}

En la dimensión análisis de perspectivas y argumentación, hubo un cambio positivo y significativo del 36.6\% $(n=15)$ en una de las tres preguntas que componen esta dimensión relacionada con el reintegro de campesinos

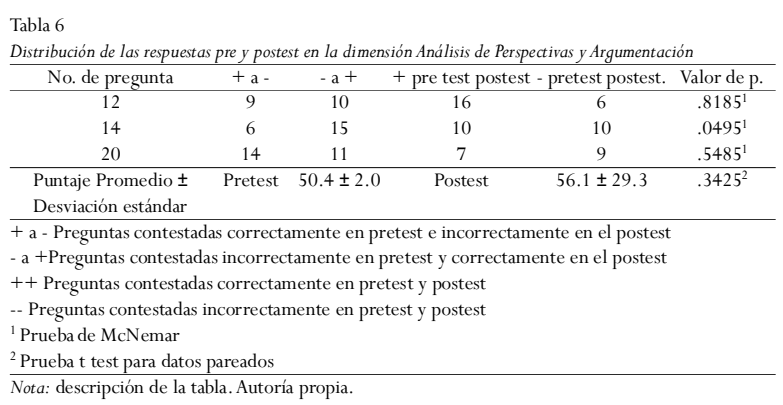


desplazados en un ecosistema humedal. No hubo diferencias estadísticamente significativas en el pre y postest de esta dimensión (Tabla 6. Figura 1).

En la dimensión Ética, no hubo un cambio significativo en el puntaje de ninguna de las preguntas que compone esta dimensión. En concordancia, no se encontraron cambios significativos en el puntaje de esta dimensión. (Table 7. Figura 1)

\begin{tabular}{|c|c|c|c|c|c|}
\hline \multicolumn{6}{|c|}{ Distribución de las respuestas pre y postest en la dimensión Ética } \\
\hline No. de pregunta & $+a-$ & $-a+$ & ++ & -. & Valor de p. \\
\hline 3 & 6 & 5 & 30 & 0 & $1.0000^{1}$ \\
\hline 18 & 4 & 17 & 8 & 12 & $.0720^{1}$ \\
\hline 19 & 13 & 6 & 22 & 0 & $.1671^{1}$ \\
\hline 23 & 7 & 9 & 1 & 24 & $.8036^{1}$ \\
\hline 24 & 6 & 10 & 21 & 4 & $.4545^{1}$ \\
\hline \multicolumn{5}{|l|}{ Desviación estándar } & $.2259^{2}$ \\
\hline $\begin{array}{l}+ \text { + a - Preguntas conte } \\
-\mathrm{a} \text { +Preguntas conte } \\
++ \text { Preguntas contes } \\
\text { - Preguntas contesta } \\
{ }^{1} \text { Prueba Exacta de M } \\
{ }^{2} \text { Prueba t-test para }\end{array}$ & 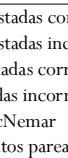 & $\begin{array}{l}\text { ectamente e } \\
\text { rrectamente } \\
\text { ctamente en } \\
\text { ctamente en } \\
\text { los }\end{array}$ & $\begin{array}{l}\text { retest e i } \\
\text { pretest y } \\
\text { test y po } \\
\text { test y po }\end{array}$ & $\begin{array}{l}\text { ctamente en } \\
\text { ctamente en }\end{array}$ & $\begin{array}{l}\text { stest } \\
\text { stest }\end{array}$ \\
\hline
\end{tabular}

No se encontraron cambios estadísticamente significativos en el puntaje total entre el pre-test (60.8 \pm 12.9$)$ y post-est $(65.1 \pm 15.4)$ con un valor $p$ de .0665 . Sin embargo, al realizar el análisis por subgrupos, se encontró que aquellos que obtuvieron un puntaje igual o menor a 60 en la evaluación inicial mejoraron significativamente sus conocimientos [pretest $=51.6$ (9.9), postest $=60(15.1)$, valor $\mathrm{p}=.018]$. VerTabla 8 .

En el análisis de regresión lineal múltiple se encontró que la diferencia pre-post fue mayor en aquellos estudiantes con puntaje inicial menor de 60 puntos comparado con aquellos que obtuvieron 60 puntos o más (beta $=14.7$, CI 95\%: 3.8, 25.7). Por el contrario, aque-

\begin{tabular}{|c|c|c|c|c|c|c|}
\hline $\begin{array}{l}\text { Tabla } 8 \\
\text { Factores asociados a } \\
\text { simple (univariado) }\end{array}$ & & $\begin{array}{l}\text { ferencia pre-pos } \\
\text { Itiple (multivar }\end{array}$ & ategia ped & ́́gica en la & la plataforma Moodle & e. Regresión lineal \\
\hline \multirow[t]{2}{*}{ Características } & \multirow[t]{2}{*}{$\mathrm{n}$} & \multirow[t]{2}{*}{ Pre-test } & Post-test & \multirow[t]{2}{*}{ Valor $\mathrm{p}$} & \multirow[t]{2}{*}{$\begin{array}{c}\text { Beta (IC 95\%) } \\
\text { Univariado }\end{array}$} & \multirow[t]{2}{*}{$\begin{array}{l}\text { Beta (IC 95\%) } \\
\text { Multivariado }^{1}\end{array}$} \\
\hline & & & Edad & & & \\
\hline 19-22 años & 21 & $59.2(14.8)$ & $62.3(12.6)$ & 0.278 & Ref & Ref \\
\hline 23-38 años & 20 & $62.4(10.7)$ & $68.0(17.7)$ & 0.150 & $2.6(-6.7,11.8)$ & $8.1(-2.4,18.6)$ \\
\hline \multicolumn{7}{|c|}{ Sexo } \\
\hline Mujeres & 38 & $60.9(13.2)$ & $64.1(14.9)$ & 0.161 & Ref & Ref \\
\hline Hombres & 3 & $58.7(10.1)$ & $77.3(19.7)$ & 0.280 & $15.5(-1.7,32.7)$ & $8.0(-2.4,18.6)$ \\
\hline \multicolumn{7}{|c|}{ Estado Civil } \\
\hline Solteros/as & 37 & $61.0(12.6)$ & $65.2(15.1)$ & 0.084 & Ref & Ref \\
\hline Casado/ Unión & 4 & $59.0(18.3)$ & $64.0(19.9)$ & 0.617 & $4.2(-0.7,9.1)$ & $-11.9(-31.1,7.3)$ \\
\hline Libre/Separado & & & & & & \\
\hline \multicolumn{7}{|c|}{ Estrato Socioeconómico } \\
\hline Nivel 1 a 3 & 26 & 60.9 (11.7) & $63.5(3.1)$ & 0.414 & Ref & Ref \\
\hline Nivel 4 a 5 & 15 & $60.5(15.3)$ & $67.7(14.7)$ & 0.030 & $4.6(-5.0,14.1)$ & $6.5(-3.5,16.5)$ \\
\hline \multicolumn{7}{|c|}{ Semestre académico } \\
\hline 6-7 & 23 & $56.3(13.3)$ & $60.7(12.7)$ & 0.160 & Ref & Ref \\
\hline $8-9$ & 18 & $66.4(10.3)$ & $70.7(16.9)$ & 0.258 & $-0.13(-9.5,9.3)$ & $1.5(-8.9,12.0)$ \\
\hline \multicolumn{7}{|c|}{ Computador propio } \\
\hline No & 1 & $\mathrm{NC}$ & $\mathrm{NC}$ & $\mathrm{NC}$ & $\mathrm{NC}$ & $\mathrm{NC}$ \\
\hline $\mathrm{Si}$ & 40 & $60.5(13.0)$ & $65.0(15.6)$ & 0.060 & & \\
\hline \multicolumn{7}{|c|}{ Frecuencia semanal de uso computador (días por semana) } \\
\hline$<5$ días/semana & 11 & $56.0(15.1)$ & $64.7(21.2)$ & 0.147 & Ref & Ref \\
\hline 5-7 días/semana & 30 & $65.2(13.1)$ & $65.2(2.4)$ & 0.266 & $-6.1(-16.4,4.3)-$ & $-14.2(-27.9,-0.5)$ \\
\hline \multicolumn{7}{|c|}{ Tiempo diario frente a un computador } \\
\hline $1-5$ horas & 20 & $57.4(12.3)$ & $62.0(3.8)$ & 0.227 & Ref & Ref \\
\hline$>5$ horas & 21 & $64.0(13.0)$ & $68.0(13.1)$ & 0.171 & $-0.6(-9.9,8.7)$ & $11.8(-0.2,23.8)$ \\
\hline \multicolumn{7}{|c|}{ Uso de la plataforma MOODLE en otras asignaturas } \\
\hline No & 5 & $64.0(14.1)$ & $70.4(5.4)$ & 0.356 & Ref & Ref \\
\hline $\mathrm{Si}$ & 36 & $60.3(12.9)$ & $64.3(16.2)$ & 0.115 & $-2.4(-16.6,11.8)$ & )$-6.5(-21.2,8.2)$ \\
\hline \multicolumn{7}{|c|}{ Puntaje inicial } \\
\hline$<=60$ & 22 & $51.6(9.9)$ & $60(15.1)$ & 0.018 & $8.8(-0.1,17.7)$ & $14.7(3.8,25.7)$ \\
\hline$>60$ & 19 & $71.4(6.1)$ & $70.9(13.8)$ & 0.885 & Ref & Ref \\
\hline
\end{tabular}

llos que usaron el computador 5-7 días por semana obtuvieron una diferencia pre-post significativamente menor que aquellos que usaron el computador menos de 5 días por semana (beta-14.2, IC 95\%: -27.9, -0.5). (Ver Tabla 8).

En conclusión, hubo cambios positivos en ocho de las 25 preguntas sobre competencias ciudadanas. Las ocho preguntas correspondían a las dimensiones Multiperspectivismo $(\mathrm{n}=3)$, Constitución Política $(\mathrm{n}=2)$, pensamiento sistémico y reflexivo $(\mathrm{n}=2)$, y análisis de Perspectivas y Argumentación ( $\mathrm{n}=1)$. Adicionalmente, en la comparación de los puntajes pre-post intervención por dimensión, solo la dimensión Multiperspectivismo mostró que el trabajo con Moodle mejoró significativamente el puntaje de conocimiento de competencias ciudadanas.

\section{Discusión}

La estrategia pedagógica aplicada en este estudio incluyó actividades implementadas en el Aula Extendida Moodle tales como foros, mapas conceptuales, cuadros comparativos y ensayos que estuvieron activos durante10 semanas en la plataforma, con el fin de reforzar los conocimientos en competencias ciudadanas adquiridos en el proceso de formación de los estudiantes.

Lamas, Massié y Quero (2010), realizaron un estudio en 222 estudiantes universitarios de Argentina sobre el uso del ambiente virtual de aprendizaje Moodle; los autores concluyeron que aunque puede ser motivante la implementación de las asignaturas en un medio tecnológico, ésta no genera impactos por sí misma, ya que necesita el compromiso de estudiantes para su propio aprendizaje, así como el diseño y la implementación consciente del profesor en las actividades, influyendo también la preferencia del estudiante por un tema específico desarrollado por medio de la plataforma. En este sentido, el estudio reportado por Pérez y Rodríguez (2016) sobre la percepción de los docentes y estudiantes para la utilización de las herramientas digitales, encontró un grado insuficiente en la percepción para el almacenamiento de información digital $(69,8 \%)$, colaborar en equipo en canales digitales $(63,4 \%)$, compartir recursos a través de herramientas en línea $(49,2 \%)$, editar y elaborar recursos $(55,5 \%)$, tener nociones informáticas $(58,6 \%)$, utilizar derechos de propiedad $(66,7 \%)$ o elegir software apropiado $(63,5 \%)$, lo cual sugiere que en efecto la motivación de docentes y estudiantes podría repercutir en la adherencia del uso de estos ambientes virtuales. 
Las instituciones educativas no son los únicos espacios apropiados para la formación de las competencias ciudadanas, ya que se debe propiciar también en la familia, los medios de comunicación y en otros espacios de socialización y participación ciudadana. Sin embargo, las universidades son pequeñas sociedades en las que los estudiantes pueden poner en práctica las competencias ciudadanas que estén aprendiendo, porque se hace necesaria la puesta en práctica de lo aprendido para solucionar problemáticas en los contextos reales y académicos. (Chaux, 2004; Betancourt, Bernate, Fonseca, \& Rodríguez, 2020). En coherencia con lo anterior, en esta investigación se encontraron cambios positivos significativos para las funciones de los representantes en la cámara de Representantes del Departamento, adopción de niños por parejas del mismo sexo, limitación diaria del número de carros particulares a circular, el proceso de configuración histórica del sistema político colombiano y el trato desventajoso de los afroamericanos; demostrando que la interacción docente-estudiante-conocimiento propiciada en el entorno de las instituciones de educación superior influyen positivamente en el desarrollo de estas competencias.

Participaron en este estudio 41 estudiantes de los cursos o asignaturas de prácticas formativas según la malla curricular o plan de estudio del programa de fisioterapia; en cuanto a las características sociodemográficas las edades estaban comprendidas entre 19 a 30 años, perteneciendo al sexo femenino el 92.85\% ; las demás variables generales evaluadas permiten conocer la población estudiantil en términos de acceso a servicios y equipos informáticos y de tecnología de la información, con el fin de determinar a futuro posibles estrategias que permitan continuar implementando la plataforma en todos los cursos o asignaturas del plan de estudios.

De estas variables se resalta que el $88.1 \%$ ha utilizado la plataforma Moodle en asignaturas previas, posiblemente esto último facilitó que todos los estudiantes realizarán la totalidad de las actividades pedagógicas implementadas en el aula extendida, al encontrarse familiarizados con la misma. Estos hallazgos contrastan con los publicados por Oproiu (2015), en donde el 45\% de los estudiantes no la usaban en todos los cursos o asignaturas, porcentaje considerado alto por el autor teniendo en cuenta que la mayoría de los encuestados ya tenían un año estudiando en la universidad; el 28\% de los que respondieron usaban la plataforma esporádicamente, es decir una vez por semana, y el 18\% una vez al mes.

Respecto a los resultados del conocimiento antes y después de la aplicación de la estrategia, al realizar el análisis por subtemas según las habilidades a desarrollar, se encontraron diferencias significativas en las preguntas relacionadas con Poder Público y con las funciones de los representantes en la Cámara de representantes del Departamento (valor de p $<.0005$ y .0042) respectivamente. Para Zuta, Velasco y Rodríguez (2014), es importante dar a conocer a los jóvenes la relevancia de la Constitución Política y las funciones de sus representantes, ya que esta nos rige como ciudadanos con derechos y deberes y nos orienta sobre las leyes que deben gobernar a toda persona dentro de una sociedad.

Para las preguntas relacionadas con el tema de Multiperspectivismo, donde el estudiante debe tener la capacidad de reconocer la existencia de diferentes perspectivas en situaciones en las que interactúan diversas partes, tres de las cuatro preguntas tuvieron diferencias estadísticamente significativas, (valor de $\mathrm{p}<$.005), lo cual podría atribuirse a que la estrategia aplicada incluyó cuatro tipos diferentes de actividades pedagógicas que pretendían propiciar un diálogo de saberes y espacios de discusión colectiva que permiten contrastar diferentes argumentos. Diversos autores señalan la importancia de esta habilidad dentro de las competencias ciudadanas, ya que es una herramienta de pensamiento para enfrentar problemas sociales, en las que también están incluidas la valoración de argumento y pensamiento sistémico. (Peña, 2013).

En cuanto al pensamiento sistémico y reflexivo, se observó un cambio positivo en relación con el proceso de configuración histórica del sistema político colombiano $(46.3 \% \mathrm{n}=19)$ y el trato desventajoso de los afroamericanos $(41.5 \%, \mathrm{n}=17)$; sin embargo, no hubo cambios estadísticamente significativos $(\mathrm{p}<.005)$ después de la intervención para toda la dimensión. En congruencia, Morales 2016) en su estudio sobre competencias solidarias en universitarios, expresa que la formación desde un punto de vista personal, comunitario y la capacidad para afrontar situaciones personales y sociales complejas con soluciones razonadas, requieren de una práctica educativa adecuada, planificada y sistemática, además de otros elementos de carácter formativo. El enfoque constructivista en los procesos de enseñanza aprendizaje que permiten estos ambientes virtuales, por medio de una interacción dialéctica entre el docente y los estudiantes en la construcción significativa del conocimiento, podría relacionarse con el cambio positivo encontrado en dos aspectos de esta dimensión dado el diálogo de saberes que permite esta plataforma; tenien- 
do en cuenta también el contexto específico de las condiciones biológicas, psicológicas, sociales, económicas, culturales y políticas que puede incidir en ambos actores del proceso educativo. (Ortiz, 2015).

Con respecto al comportamiento de las preguntas de análisis de perspectivas y argumentación, es importante resaltar que hubo cambios significativos en la pregunta relacionada con el proceso de configuración histórica del sistema político colombiano (p .0026). Para las preguntas de lectura crítica y argumentación no se encontraron cambios positivos en el postest, lo que puede asociarse probablemente a las dificultades que presentan los estudiantes al ingreso y durante la formación de pregrado en las habilidades relacionadas con lectura crítica, redacción, comprensión de lectura y capacidad de síntesis, de igual manera, el número de preguntas que evalúa esta competencia son muy pocas, por lo que se sugiere realizar futuros estudios incluyendo más preguntas que permitan evaluar esta dimensión en aras de encontrar diferencias significativas.

Respecto a las preguntas relacionadas directamente con Ética, no hubo diferencias significativas en ninguna de los aspectos de esta dimensión, estos resultados permiten inferir que si bien es cierto que la política educativa del Ministerio de Educación Nacional del año 2010, se fundamenta en promover una educación de calidad que forme mejores seres humanos, ciudadanos con valores éticos, respetuosos de lo público, que ejercen los derechos humanos, cumplen con sus responsabilidades sociales y conviven en paz, debe haber un mayor compromiso de las universidades en involucrar estas cátedras en sus planes de estudio.

Algunos autores como Trianes y Fernández (2010), afirman que las competencias ciudadanas, son valores solidarios que deben constituirse en el principio rector de toda educación, con el fin de lograr una formación integral del estudiante, promoviendo actitudes y valores éticos que se verán reflejados en la vida diaria. En concordancia, Zuta, et at. (2014), refieren que una educación ciudadana permite a la persona involucrarse y ser partícipe en el mejoramiento de las condiciones de vida personal y de su entorno cercano y global, permiten igualmente consolidar y fortalecer la democracia como estilo de vida y como sistema, posibilitando así convivir en sociedad en forma pacífica.

Los resultados de este estudio no mostraron diferencias estadísticamente significativas en los conocimientos de los estudiantes sobre las competencias ciudadanas antes y después del uso de la plataforma Moodle respecto al puntaje total para el pre-test y post-est; no obstante, la categorización por subgrupos si mostró cambios significativos para el grupo menor a 60 puntos en el análisis por regresión lineal múltiple. En oposición a estos hallazgos, Iglesias, Ruiz y Aguido (2015) realizaron un estudio para evaluar el desarrollo de las competencias de trabajo en equipo y compromiso, teniendo en cuenta las interacciones de los estudiantes en la plataforma Moodle; si bien las variables usadas por los autores difieren con las aplicadas en esta investigación, el estudio también evalúa el uso de la plataforma Moodle en la adquisición de competencias en los educandos. Los resultados de la regresión múltiple reportados por los autores no mostraron diferencias significativas en la adquisición de las competencias evaluadas en relación con las interacciones de los estudiantes en la plataforma.

Por último, cabe mencionar que la herramienta tecnológica utilizada en este estudio para la aplicación de la estrategia pedagógica complementa el trabajo independiente de los estudiantes, permitiendo por medio de la virtualidad el desarrollo de las tareas con constante seguimiento de los docentes, teniendo en cuenta que éstos son cursos o asignaturas presenciales, en los cuales las horas de acompañamiento directo se dedican principalmente a la orientación de los contenidos disciplinares. Así mismo, los resultados derivados de esta investigación muestran que el uso de las aulas extendidas en la formación de los estudiantes favorece y complementa la adquisición de algunos aspectos en las competencias, a su vez podrían nutrir las discusiones y análisis curriculares al interior de las universidades dado que permite retroalimentar las estrategias institucionales que buscan mejorar el rendimiento de los estudiantes en las Pruebas de Estado, donde se evalúan también estas competencias en el país.

Dentro de las limitaciones de este estudio cabe mencionar que la capacitación para el uso adecuado de la plataforma como aula extendida fue de varias semanas lo que atrasó un poco el desarrollo de la estrategia pedagógica por parte de los estudiantes. De otra parte, el análisis general de los resultados antes y después de la ejecución de las actividades en la plataforma, muestran que no se alcanzaron cambios significativos en los conocimientos con relación a algunas dimensiones; lo cual puede estar asociado a la probable presencia de sesgo de medición ya que no se realizó evaluación de las propiedades psicométricas del cuestionario. Así mismo, el cambio de metodología de aplicación de la evaluación de formato papel como se hacía tradicionalmente, al formato virtual pudo afectar las respuestas de los participantes. 


\section{Conclusión}

Los resultados mostraron que una intervención educativa con Moodle mejoró el conocimiento de algunas competencias ciudadanas entre los estudiantes de fisioterapia, especialmente en la dimensión de multiperspectivismo.

No hubo cambios significativos en la dimensión de Ética al ser evaluada en los estudiantes, lo que lleva a inferir que se debe seguir implementando y fortaleciendo esta dimensión desde el Ministerio de Educación y desde las Instituciones de Educación Superior que son los entes comprometidos en la formación universitaria.

Se empleó una estrategia pedagógica con cuatro actividades que favorecieron el aprendizaje significativo de los estudiantes en competencias ciudadanas, siendo estas actividades, Foro, Mapa Conceptual, Cuadro Comparativo y Ensayo, desarrollando en el estudiante el pensamiento crítico, la metacognición y la capacidad de búsqueda rigurosa de información y la habilidad de la comunicación escrita.

Es importante que las universidades generen espacios de discusión académica, con el fin de mejorar las estrategias institucionales que permitan transversalizar la formación integral en competencias ciudadanas dentro de los planes de estudios de todos los programas.

\section{Declaración de Conflicto de Interés}

Los autores declaran no tener conflicto de interés alguno.

\section{Referencias}

Arancibia, V., Herrera, P., \& Strasser, K. (1999). Manual de Psicología Educacional. México: Alfaomega.

Asamblea Nacional Constituyente. (1991). Constitución Política de La República de Colombia. Bogotá D.E.: Asamblea Nacional Constituyente.

Betancourt, M., Bernate, J., Fonseca, I., \& Rodríguez, L. (2020). Revisión documental de estrategias pedagógicas utilizadas en el área de la educación física, para fortalecer las competencias ciudadanas. Retos, 38(38), 845851. DOI: https://doi.org/10.47197/ retos.v38i38.74918

Castillo Díaz, M., Larios Rosillo, V., \& García Ponce de Leon, O. (2010). Percepción de los docentes de la utilización de las Tecnologías de la Información y la Comunicación. Revista lberoamericana de Educación. 1-10.

Chaparro Peláez, J., Iglesias Prada, S., \& Pascual Miguel, F.
(2010). Uso del registro de actividad de Moodle para un estudio del rendimiento académico de alumnos en entornos en línea y presencial. In 4th International Conference on Industrial Engineering and Industrial Management, 753-760.

Chaux, E. (27 de marzo de 2004). ¿Qué son las competencias ciudadanas? . Obtenido de Semana. Recuperadode: https:/ /www.semana.com/on-line/articulo/que-competencias-ciudadanas / 64379-3

Cordero Torres, J.A., \& Caballero Oliver, A. (2015). La plataforma Moodle: Una herramienta útil para la formación en soporte vital.Análisis de las encuestas de Satisfacción a los alumnos e instructores de los cursos de soporte vital avanzado del programa ESVAP de la semFYC. Atención Primaria, 47(6), 376-384. doi:10.1016/j.aprim.2015.02.006

Costa, C., Alvelos, H., \& Teixeira, L. (2012). The use of Moodle e-learning platform: a study in a Portuguese University. Procedia Technology (5), 334-343 doi:https: / / doi.org/10.1016/j.protcy.2012.09.037

De La Rosa Ríos, J.C. (2011). Aplicación de la plataforma moodle para mejorar el rendimiento académico en la enseñanza de la asignatura de cultura de la calidad total en la Facultad de Administración de la Universidad del Callao. Tesis de posgrado. Universidad Nacional Mayor De San Marcos, Lima, Perú. Fernández Miranda, M., \& Bermúdez Torres, M.A. (2009). La plataforma virtual como estrategia para mejorar el rendimiento escolar de los alumnos en la I.E.P Coronel José Joaquín Inclán de Piura. Revista Digital Sociedad de la Información. 15, 1-19.

Fernández, A. (2009). Las plataformas e-learning para la enseñanza y el aprendizaje universitario en internet. Universidad Complutense de Madrid: https://eprints.ucm.es/ 10682/1/capituloE_learning.pdf

García Cabrero, B., \& Pineda Ortega,V. J. (2010). La construcción de conocimiento en foros virtuales de discusión entre pares. Revista mexicana de investigación educativa, 15(44), 85-111

Gros Salvat, B., \& Contreras, D. (2006). La alfabetización digital y el desarrollo de competencias ciudadanas Revista Iberoamericana de Educación, (49). doi:http:// hdl.handle.net/2445/57176

Hobsbawm, E. (1999). Historia del Siglo XX. Buenos Aires, Argentina: Crítica Grijalbo Mondadori.

Iglesias Pradas, S., Ruiz de Azcárate, C., \& Agudo Peregrina, Á. F. (2015). Assessing the suitability of student interactions from Moodle data logs as predictors of crosscurricular competencies. Computers in Human Behavior, 47, 81-89. https://doi.org/10.1016/ j.chb.2014.09.065

Instituto Colombiano para Evaluación de la Educación Icfes. 
(2018). Consulta de Resultados Competencias Genéricas Pruebas Saber Pro. ICFES: http://www.icfes.gov.co/resultados/saber-pro-resultados-individuales/resultados-agregados-saber-pro/competencias-genéricas

Instituto Colombiano para la Evaluación de la Educación Superior (ICFES). (2015). Módulo de Competencias Ciudadanas. https: / /www.icfes.gov.co/documents/20143/ 493891/Competencias\%20ciudadanas\%202015-1.pdf

Instituto Colombiano para la Evaluación de la Educación Superior Icfes. (2015). Sistema Nacional de Evaluación estandarizada de la Educación. : https: / /www.icfes.gov.co/ d o c u m e n t s / $20143 / 193784$ / Alineacion\%20examen\%20Saber\%2011.pdf

Lamas, M.L., Massié, A.I, \& Quero, E.D. (2010). Implementación de un aula virtual bajo la modalidad mixta: El Caso de Química Agrícola en la Universidad Nacional de Salta. Formación Universitaria 3(4), 3-12. doi:http: / / dx.doi.org/10.4067/S071850062010000400002

Maldonado Berea, G.A, \& Vega Gea, E. (2015). Actitud de los estudiantes universitarios ante la plataforma moodle. Píxel-Bit. Revista de Medios y Educación, (47), 105-117. . doi:10.12795/pixelbit.2015.i47.07

Manterola, C., \& Otzen, T. (2015). Estudios Experimentales 2 Parte. Estudios Cuasi-Experimentales. International Journal of Morphology, 33(1), 382-387:http://dx.doi.org/ 10.4067/S0717-95022015000100060

Martín Blas, T., \& Serrano Fernández, A. (2009). The role of new technologies in the learning process: Moodle as a teaching tool in Physics. Computadoras y Educación, 52(1). doi:https: / /doi.org/10.1016/j.compedu.2008.06.005

Mieles Barrera, M.D, \& Alvarado Salgado, S.V. (2012). Ciudadanías y competencias ciudadanas. Instituto de Estudios Políticos, (40), 53-75.

Ministerio de Comunicaciones de Colombia. (2008). Plan Nacional de Tecnologías de la Información y las Comunicaciones 2008-2019. https://www.mintic.gov.co/portal/604/ articles-8247_pe_plan_tic_colombia_2009_2018.pdf

Ministerio de Educación Nacional de Colombia. (2004). Competencias ciudadanas Habilidades para saber vivir en paz. Altablero. https: / / www.mineducacion.gov.co/1621/ articles-99165_archivo_pdf_Lineamientos.pdf

Ministerio de Educación Nacional de Colombia. (2006). Estándares básicos de Competencias Ciudadanas. https: / / www. mineducacion.gov.co/1621/articles116042_archivo_pdf4.pdf

Ministerio de Educación Nacional República de Colombia, Resultados Pruebas ICFES. 2014

Ministerio de Educación Nacional República de Colombia. (2010). Lineamientos para la articulación de la educación de la edad media. https: / /www.mineducacion.gov.co/1621/
articles-99165_archivo_pdf_Lineamientos.pdf

Montes, N., \& Machado, E. (2011). Estrategias docentes y métodos de enseñanza-aprendizaje en la Educación Superior. Humanidades Médicas, 11(3), 475-488.

Morales Rodríguez, F.M. (2016). Evaluación mediante rúbrica de la adquisición de competencias solidarias en universitarios. Revista de Educación Mediática y TIC. EDMETIC. 5(1), 93-112. doi:https://doi.org/10.21071/ edmetic.v5i1.4018

Nass, L., \& Merino, J. (2008). El experimento natural como un nuevo diseño cuasi-experimental en investigación social y de salud. Revista Ciencia y Enfermería, 14(2). doi:http: / / dx.doi.org/10.4067/S071795532008000200002

Oproiu, G. (2015).A Study about Using E-learning Platform (Moodle) in University Teaching Process. Procedia - Social and Behavioral Sciences, 180, 426-432. DOI:10.1016/ j.sbspro.2015.02.140

Ortí, C. B. (2011). Las tecnologías de la información y comunicación(TIC). Univ.Val., UnidadTecnol. Educ,(951), 1-7.

Peña, M. (2013). Evaluación en Competencias ciudadanas en Colombia. Bogotá D.C.: Instituto Colombiano para la Evaluación de la Educación.

Pérez Casales, R., Rojas Castro, J., \& Paulí Hechavarría, G. (2008). Algunas experiencias didácticas en el entorno de la plataforma Moodle. Revista de Informática Educativa y Medios Audiovisuales, 5(19), 1-10.

Pérez Escoda,A., \& Rodríguez Conde, M.J. (2016). Evaluación de las competencias digitales autopercibidas del profesorado de Educación Primaria en Castilla y León (España). Revista de Investigación Educativa, 34(2), 399 415. doi:https://doi.org/10.6018/rie.34.2.215121

Pérez, R., Álvarez, E., García, M. S., Pascual, M., \& Fombona, J. (2004). Foro Virtual: sus límites y posibilidades en el proceso de enseñanza-aprendizaje. Edutec

Pimienta, J. (2008). Constructivismo: Estrategias para aprender a aprender. México: Pearson.

Trianes, M., \& Fernández, C. (2001). Aprender a ser personas y a convivir. Un programa para secundaria. Bilbao: Desclée de Brouwer.

Universidad de Santander. (2014). Procedimiento de Creación, Implementación de aulas extendidas con plataforma Moodle posgrados. Ambientes Virtuales de Aprendizaje. Bucaramanga, Santander: AVA Moodle. UDES. .

Valenzuela Zambrano, B., \& Pérez Villalobos, M. (2013). Aprendizaje autorregulado a través de la plataforma virtual Moodle. Educación y Educadores, 16(1), 66-79.

Zuta, E., \& Velasco, A. R. (2014). Desarrollo de competencias ciudadanas mediante un curso socialmente responsable. Educación, 23(45), 51-66 